\title{
MOTOR DISTURBANCES IN LETHARGIC ENCEPHALITIS *
}

\author{
ISADOR ABRAHAMSON, M.D.
}

Associate Neurologist, Mount Sinai Hospital; Visiting Neurologist; Montefiore Home

NEW YORK

We have seen that the main stress of the toxemia of lethargic encephalitis falls on the nerve cells. At certain sites the toxic action is reinforced by the direct attack of the infectious agents (LoewyStrauss bodies?) on the nerve cells. The nerve sites attacked by these organisms are determined in part by the particular mucous membrane in which the initial invasion takes place. From this area, the organisms are carried along the lymphatics to the central nervous system. As the habitually invaded area is the nasopharyngeal mucosa, the nerve sites usually attacked are in the head end of the nerve axis; but if the intestinal mucosa be the invaded area, the brunt of the attack falls on the caudal end of the nerve axis. The direction, extent and severity of the attack on the nervous system determine the nature and degree of the resulting functional disturbances. It has already been stated that the more extensive the nervous mechanism on which a function depends, the more inevitable is the implication of that function in the attack. The nervous mechanism that subserves the function of movement is coextensive with the nervous system. In lethargic encephalitis movement is invariably deranged.

Lethargic encephalitis may affect the function of movement in all predictable ways, to all conceivable degrees and at all possible points of the motor mechanism. In different epidemics, and even during the same epidemic in different localities, or at different periods, particular varieties of motor derangement tend to prevail. In spite of such episodal variations among the qualities of the motor defect of lethargic encephalitis are certain that are banal and certain that at least in their emphasis are distinctive and pathognomonic.

Of any two movements, that which depends on the more extensive nerve mechanism is-ceteris paribus - the more liable to derangement in this infection. Where separate paths convey habitually correlated impulses to an associated group of muscles, sudden interference with any one path may disorganize temporarily the whole function. The

* Read at the Stated Meeting on Lethargic Encephalitis, at the New York Academy of Medicine, May 20, 1920. 
initial result of the lesion may, therefore, be wholly disproportionate to its cause; and this disproportion may be accentuated if, as in the eye, the impairment of movement deranges another and more sensitive function.

\section{MOTOR SYMPTOMS}

Motor Disturbance of Eye.-The widespread nerve supply of the motor mechanism of the eye seldom wholly escapes implication in lethargic encephalitis. Double vision, near vision, blurred vision and other perceptual consequences may signify such motor implication even before it is detectable by skilled medical observation. The alternative paths of nerve impulses to the muscles of the eye may permit the speedy compensation of this functional disturbance. Hence, patients frequently neglect to mention it among their symptoms, unless specifically questioned regarding it.

In addition to these transient symptoms, lasting signs appear of impairment of the motor mechanism of the eyes. As a rule, the eyelids droop, first one, then the other. The droop is not commonly equal on both sides, and seldom completely closes both eyes; usually the palpebral fissures persist, one narrower than the other. In most cases the eyelids can be freely raised at will; but they fall again. Sometimes, however, they are not raised; but the patient then seems rarely to exert himself to open his eyes-the forehead is not wrinkled by his efforts.

The eyes assume the "rest" position; their axes being directed straight forward. They can be rotated as a rule into any normal position; but they do not maintain it; they tend to swing back to the middle line. Internal convergence is usually possible for a short time, at the end of which one eye rotates out again. The eyes may move deliberately and by stages, moving and halting and moving again, then swinging back to the position from which they started. Sometimes this movement is definitely cog-wheel, like the eye movement seen in Parkinson's disease. Infrequently, definite but seldom conspicuous squints occur.

The pupillary reaction to light and accommodation may be lost or diminished. Usually both are unaffected. The pupils under a constant intensity of illumination contract and dilate, unable to maintain the pupillary posture corresponding to the illumination. A similar instability may be observable in accommodation.

Facial Muscles.-The facial muscles are often affected. Spasms, tics and fibrillation may sometimes be seen in them and occasionally nuclear paralysis. As a rule the facial affection consists chiefly of a loss of tone, a flattening of the muscles, with a fading of the wrinkles, and a lessening of the habitual facial folds. This tone 
loss begins on one side and then spreads to the other. It is usually not complete or symmetrical; on one side it may affect the upper third, on the other the lower two thirds. It tends to disappear in emotional expression. It suggests a supranuclear palsy, but the apparent weakness may vanish in whole or in part on voluntary movement. Occasionally, even in patients who are not extremely apathetic, a partial or complete absence of emotional expression is observable in a face that otherwise shows no detectable motor defect. The facial weakness, no matter what its type may be, is seldom sufficient to lead to lacrimation, to drooling or to interference even with mastication.

Fifth Nerve.-Occasionally, the motor root of the fifth nerve suffers. During the irritative stage, chewing or grinding movements occur, and later lock-jaw may supervene. Even without premonitory movements, the jaw muscles may gradually stiffen till the mouth scarcely can be forced open. This irritative stage may be followed by a typical paralytic condition.

Tongue.-The tongue is implicated almost as frequently as the face. After a period of fibrillary twitching, the tongue on protrusion deviates to the weakened side; but this deviation bears no regular correspondence to any coexisting facial palsy, except in banal cases of hemiplegia. Later, the tongue is protruded with increasing difficulty or lies an inert or tremulous mass, immovable on the floor of the mouth. In such instances mastication and speech suffer.

The Soft Palate and the Constrictors of the Pharynx.-These may be so weakened as to render swallowing difficult or even impossible and to necessitate feeding by the stomach tube.

Cranial Motor Nerves.-Of the cranial motor nerves the eleventh (spinal accessory) is least commonly affected. When it is attacked, there may be wry neck but more frequently both sides are equally involved. Associated with the spasms, fibrillation, or loss of tone of the trapezeii and sternomastoids are similar affections of the other muscles of the neck region. Cephaloptosis or cephaloplegia, similar to that seen in poliomyelitis, is occasionally observed in lethargic encephalitis, especially in children.

As the cranial motor nerves are affected, in like manner the disease attacks the spinal motor nerves; and the consequences observed in the muscles of the head and neck are mutatis mutandi, observed in the muscles of the limbs and trunk. In the affected muscles spasms, fibrillations, weakness and loss of tone usually occur. The weakness may follow the spasms but oftener develops independently. Foot drop, wrist drop, sagging shoulder and other abnormal attitudes are assumed in the "resting" position. The dropped foot, wrist or shoulder, as a rule, can be raised at will; but it quickly drops again. 
Hemiplegias and Monoplegias.-Not rarely banal hemiplegias or monoplegias occur. In these the distal muscles of the extremities are more involved than the proximal. Some power of movement usually persists in the trunk muscles of the affected side and in the muscles nearest the trunk in the affected limbs. The affected muscles are rigid and immobile. They assume the characteristic hemiplegic attitude, which is determined mainly by the dominance of the flexors over the extensors. Occasionally athetoid movements are seen in the paralyzed limbs.

Besides this banal hemiplegia, rigidity and loss of power of lesser degree occur, often hemiplegic in distribution. The rigid muscles, partially immobilized, tend to assume the typical hemiplegic posture and tremors appear in them. The characteristic tremor in lethargic encephalitis, like the tremor of Parkinson's disease, visibly affects extremities more than the head and trunk and is exaggerated by emotional excitement and by indirect intention. The lethargic encephalitis tremor, however, is not present "at rest"; it is markedly increased by direct intention and grows in amplitude as the objective of the movement is approached; it involves the forearm and wrist more than the hand and finger joints and the leg and ankle more than the toes; it is coarser, less symmetrical and more irregular than the typical Parkinsonian tremor. Where such a tremor ends, and a rhythmic movement purposeful in form but not in intent, begins, is difficult to define. The coarse pseudoparkinsonian tremor of lethargic encephalitis is scarcely distinguishable from a slight, irregular rhythmic purposeful movement.

In addition to this coarse tremor, the small, rapid tremor seen in most acute infectious diseases, occurs also in lethargic encephalitis.

Occasionally epilepsy develops.

The most characteristic of the motor disturbances is instability of posture.

\section{LOSS OF MUSCLE TONE AND ITS RELATION TO POSTURE}

Posture is the expression not of one, but of all the influences that determine muscle tone. The fundamental influence is the nutrition of the individual muscle cell, which is dependent partly on general nutritive conditions and partly on local and general control of these conditions through reflex and chemical action. In lethargic encephalitis loss of muscle tone occurs.

Part of this loss is analogous to that which occurs in all acute infectious diseases. Fever, toxemia, and their concomitant endocrine disturbances always reduce muscle tone. With the development of acute infectious fevers reflexes, such as the knee jerks, which depend on muscle tone, diminish or disappear; with the abatement of the 
infection the muscle tone returns and the reflexes related to it recover their activity. Among infectious fevers lethargic encephalitis is conspicuous by its power directly to lower muscle tone, perhaps because of the virulence of its toxins, perhaps because of their inhibitory action on the pituitary and suprarenal glands.

Part of the loss may be due to inflammatory implication of nerve centers and paths by which the tone of the musculature is regulated. In lethargic encephalitis lesions may occur in the vestibular and cerebellar systems through which local, sectional and general changes in muscle tone are initiated and correlated. But the tone loss is common and such lesions are infrequent.

Part of this loss may be due also to the injury or destruction of the lower motor neuron, either at its origin in the nuclei of the anterior horn of the spinal cord and in their pontile and medullary analogues, or during its course through the peripheral motor nerve. A preliminary stage of irritation associated with muscular rigidity and fibrillation and myoclonia sometimes may be observed; then atonicity, atrophy and quantitative or qualitative changes in the electrical reactions occur. This loss is seldom widespread. It does occur, but it is often absent. Habitually, the electrical responses to faradism and to galvanism are unaltered. These responses are given by the fibrillar portion of voluntary muscle; which is therefore not blamable for the customary loss of muscle tone in lethargic encephalitis.

The tone loss is evident in the afflicted muscles even in so-called "rest" postures. There is in life no such thing as a posture of absolute "rest." "Rest" is a purely relative term. It signifies usually a posture of mental repose, the posture that is determined chiefly by the action of gravity. Its maintenance requires definite degrees of muscle tone. Hence, the afflicted facial muscles "at rest" flatten, the wrinkles fade and the usual facial folds disappear. In other words, owing to depreciation of muscle tone, the normal facial posture "at rest" is lost.

When a muscle contracts its tone increases. The increase of tone that accompanies voluntary movement may be adequate to mask the tone loss of the "resting" muscle. Thus, the atonic facial muscle sometimes moves at will with seeming perfection; but sometimes the flattening persists. The amount of disturbance persisting in movement measures the failure of the increased tonicity of the voluntary moving muscles to mask the tone loss of the "resting" muscle.

In moving, a muscle proceeds from an existing attitude through a series of intermediate postures to the desired attitude. To every posture pertains a definite muscular tone. Throughout a movement the tone of each posture must be maintained until that of its successor in the movement can be superimposed. Otherwise the desired 
attitude is not directly attained and the movement is oscillatory or jerky. Lack of tone inherent to the intermediate postures leads to the phenomena seen when the eyes move-the slow rotations by stages and the persistent tendency to swing back to the "rest" position. It is as if the increased tone excited by the voluntary movement sufficed to attain the serial posture but not to preserve it. Hence, the eye swings to and fro in its rotation toward the desired goal.

And when the desired posture is attained by the eyes, lack of the necessary tone cuts short its duration. The eyelids can be raised at will but they fall again; and as often as they fall they can be raised once more only to fall once more. On internal convergence, an eye soon swings away. It may be brought in again only to swing out once more. With a constant source of light the pupil dilates and contracts. The outstretched arms may fall and be raised at will only to fall again.

This inability to maintain posture is not a fatigue manifestation, although fatigue may magnify it. It is not comparable with the similar phenomenon seen in myasthenia gravis. It is not a paralytic phenomenon. The movements can be indefinitely repeated and the same phenomenon is seen at the beginning as at the end of the test. The muscles show neither myasthenic nor atrophic electrical reactions. The lack of power to preserve posture is not due to the general lowering of tone for it affects only individual muscles or groups in particular areas.

In any movement certain muscles, agonists, contract, and they may be aided by the simultaneous contraction of others (synergists) which act similarly. Synchronously, the antagonistic muscles actively relax. If the antagonistic muscles do not adequately relax, the contracting agonists must first overcome the pull of the unrelaxed antagonists before movement can begin; and the movement proceeds by jerks and oscillations according to the momentary dominances of the agonists or antagonists in their contest. Such a struggle for dominance may occur. The evidence of it is spasms of the antagonistic muscles. Even without appreciable spasm there, the inability to maintain posture may exist. The force of gravity may suffice to destroy the posture attained.

When a posture is achieved it is maintained against gravity mainly by virtue of the tone inherent in the sarcoplasmatic portion of volun. tary muscle. The sarcoplasmatic portion of voluntary muscle is distinguished from the fibrillar by its histologic structure, chemical composition, electrical reaction, function and nerve supply. The nerve supply is derived from neurons which originate in cells in the lateral horns of the spinal cord and their analogues in the cerebral end of the nerve axis. Through these nerves the sarcoplasmatic tone is 
maintained on which depends the posture of the muscles. In lethargic encephalitis the nerve mechanism which subserves the sarcoplasmatic portion may be implicated, giving rise to their particular form of loss of power to maintain posture. Depression of the tone of the sarcoplasmatic portion of the agonists is probably the basis of this postural fault; but the tone of the antagonistic muscles may also be unduly exalted, for although spasm may be absent, the movements may nevertheless be somewhat stiff. At present, we do not know with certainty the precise seat of the defect. Investigation of it may afford valuable data regarding the nature of the relaxation of the antagonist and its relation to sarcoplasmatic tone.

It is noteworthy that the patient sometimes makes no effort to restore the lost posture. As there is no detectable sensory loss the lack of effort is probably due to mental apathy. This postural defect may arise when the lower motor neuron is demonstrably intact. It affects postures voluntarily assumed. There must exist, therefore, not one final common path to voluntary muscle, as Sherrington teaches, but two paths, namely, the lower motor neuron, its cell in the anterior horn and its white medullated fiber passing to the voluntary muscle, and the postural nerve of voluntary muscle. In poliomyelitis the lower motor neuron is mainly and commonly affected, and the postural nerve as a rule escapes. In lethargic encephalitis the lower motor neuron as a rule escapes and the postural nerve is mainly and commonly affected. 